\title{
CONVERGENCE AND DIVERGENCE OF SERIES CONJUGATE TO A CONVERGENT MULTIPLE FOURIER SERIES
}

\author{
BY
}

\section{J. MARSHALL ASH(1) AND LAWRENCE GLUCK}

\begin{abstract}
In this note we consider to what extent the classical theorems of Plessner and Kuttner comparing the set of convergence of a trigonometric series with that of the conjugate trigonometric series can be generalized to higher dimensions. We show that if a function belongs to $L^{p}, p>1$, of the 2-torus, then the convergence (= unrestricted rectangular convergence) of the Fourier series on a set implies its three conjugate functions converge almost everywhere on that set. That this theorem approaches the best possible may be seen from two examples which show that the dimension may not be increased to 3 , nor the required power of integrability be decreased to 1 .

We also construct a continuous function having a boundedly divergent Fourier series of power series type and an a.e. circularly convergent double Fourier series whose $y$-conjugate diverges circularly a.e.

Our $L^{p}$ result depends on a theorem of $L$. Gogöladze (our proof is included for the reader's convenience), work of J. M. Ash and G. Welland on $(C, 1,0)$ summability, and on a result deducing the boundedness of certain partial linear means from convergence of those partial means. The construction of the counterexamples utilizes examples given by C. Fefferman, J. Marcinkiewicz, A. Zygmund, D. Menšov, and the present authors' earlier work.
\end{abstract}

1. Introduction. In this note our concern is with the convergence and divergence of multiple trigonometric series; primarily with the extension to higher dimensions of the classical one-dimensional theorems of Kuttner and Plessner.

THEOREM OF KUTTNER. If $T=\Sigma c_{n} \exp ($ inx $)$ converges everywhere on a measurable set $E \subset[0,2 \pi)$ and $\widetilde{T}=\Sigma-(i \operatorname{sgn} n) c_{n} \exp (i n x)$ is Cesàro summable on $E$, then $\widetilde{T}$ converges almost everywhere on $E$.

Plessner's theorem elegantly removes the summability condition.

Received by the editors November 28, 1973, and in revised form, March 22, 1974.

AMS (MOS) subject classifications (1970). Primary 42A92, 42A40, 42A20; Secondary 42A04, 42A24, 40G05, $40 \mathrm{C} 05$.

Key words and phrases. Unrestrictedly rectangularly, restrictedly rectangularly, $(C, 1,0)$ summable, Plessner's theorem, Kuttner's theorem, conjugate function, conjugate series, multiple Fourier series.

(1) Research partially supported by NSF Grant No. GP-39256. 
THEOREM OF PLESSNER. If T converges everywhere on a measurable set $E \subset[0,2 \pi)$, then $\widetilde{T}$ converges almost everywhere on $E$.

Part of the beauty of Plessner's theorem lies in its extreme generalityno restriction being placed on $T$ or $E$. In higher dimensions such sweeping generality cannot be obtained, although in this note we are able to show, for example, that if convergence is taken to mean unrestricted rectangular convergence, $\left({ }^{2}\right)$ Plessner's theorem is true on the 2-torus for the Fourier series of $L^{p}$ functions $p>1$ (Theorem 4). While at first glance this may seem a fairly puny extension of Plessner's theorem to two dimensions, it does in fact approach the best possible in two ways, since we show by counterexample that Plessner's theorem is false both for integrable functions on $T^{2}$ (Theorem 2) and for continuous functions on $T^{3}$ (Theorem 3). In doing this we also construct a continuous function having a boundedly divergent double Fourier series of power series type (Theorem 1) and an example of an a.e. circularly convergent double Fourier series whose $y$-conjugate diverges circularly a.e. (Theorem 8 ).

By contrast, Kuttner's theorem comes through to two dimensions unscathed in the form of the theorem of Gogolladze (Theorem 5) below. Since we make use of this theorem, we append our proof of it. Our positive result (two-dimensional) depends upon this theorem and a result of Ash and Welland (Theorem 6) on summability of double Fourier series, and ultimately on the Carleson-Hunt one-dimensional $L^{p}$ convergence theorem. Our negative results (counterexamples) utilize the boundedly divergent Fourier series of one-dimensional power series type of Marcinkiewicz and Zygmund and previous work of the present authors [1], and depend ultimately on Fefferman's counterexample of a continuous everywhere divergent double Fourier series. It becomes evident that the situation with regard to Plessner's theorem in higher dimensions is intimately connected with the Carleson-Hunt theorem and its nongeneralizability to higher dimension or to $L^{1}$ of the circle.

Our proof of Gogöladze's theorem requires a result (Theorem 7) of independent interest which generalizes to certain summability methods (in particular, to $(C, 1,0)$ summability) the fact that convergence of a double series implies almost everywhere the boundedness of the partial sums.

(2) This mode of convergence is defined in §2. Such convergence has some properties markedly different from ordinary convergence of one-dimensional series. For example, a convergent series need not be bounded [2, p. 406]; and as Charles Fefferman has shown with the spectacular counterexample of a continuous function on the torus whose double Fourier series is divergent everywhere [4], Carleson's famous one-dimensional theorem on the a.e. convergence of the Fourier series of an $L^{2}$ function cannot be extended to two dimensions using this definition of convergence. 
The final section consists of extensions, related results, and open questions.

We would like to express our gratitude to Professors Charles Fefferman and Antoni Zygmund for many helpful conversations.

2. Notation. Let $Z^{k}=\left\{\mathrm{x} \in R^{k} \mid\right.$ all $x_{i}$ are integers $\}$ be the integral lattice points in $k$-dimensional Euclidean space and $T^{k}=[0,2 \pi) \times[0,2 \pi) \times \ldots \times$ $[0,2 \pi)$ be $R^{k}$ with vectors $\mathrm{x}$ and $\mathrm{y}$ identified whenever $x_{i} \equiv y_{i}(\bmod 2 \pi), i=$ $1, \ldots, k$.

We shall be concerned with the formal trigonometric series $T$ where

$$
T(\mathrm{x})=\sum_{\mathrm{m} \in Z^{k}} a_{\mathrm{m}} \exp i \mathrm{~m} \cdot \mathrm{x}, \quad \mathrm{x} \in T^{k}, a_{\mathrm{m}} \in C .
$$

If $a_{\mathrm{m}}=(2 \pi)^{-k} \int_{T^{k}} H(\mathbf{x}) \exp (-i \mathrm{~m} \cdot \mathbf{x}) d \mathbf{x}$ where $H(\mathbf{x}) \in L^{1}\left(T^{k}\right)$, we say $T$ is the Fourier series of $H$ and write $T=S[H]$.

For $n \geqslant 0$, let $N(n)=\left\{\mathrm{m} \in Z^{k} \mid m_{i} \geqslant n, i=1, \ldots, k\right\}$ and for each eccentricity $E \in[1, \infty]$, let $N(n, E)=\left\{\mathrm{m} \in N(n) \mid 1 / E \leqslant m_{i} / m_{j} \leqslant E, 1 \leqslant i\right.$, $j \leqslant k\}$.

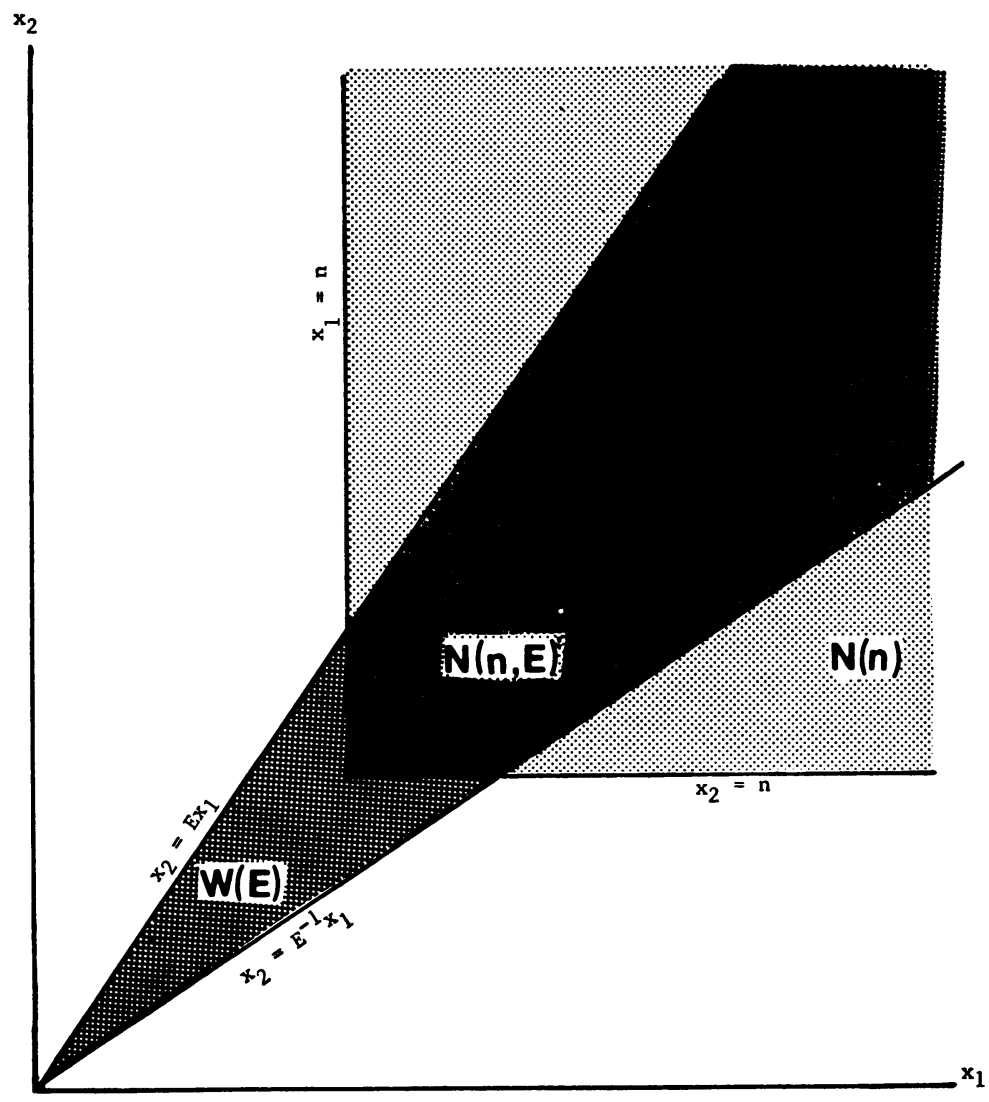

FIGURE 1 
When $k=2, N(n)$ consists of those lattice points "north and east" of $\mathbf{n}=(n, n)$, while $N(n, E)$ are those points of $N(n)$ which are also in the wedge $W(E)$ formed by the lines $x_{2}=E x_{1}$ and $x_{2}=(1 / E) x_{1}$. (See Figure 1.) Note that $N(n, 1)=\{(m, m, \ldots, m) \mid m \geqslant n\}$ and that, formally, $N(n, \infty)=N(n)$. The multiple $k$-dimensional numerical series $S=\Sigma_{\mathrm{m} \in Z^{k}} c_{\mathrm{m}}=\Sigma c_{\mathrm{m}}$ has $m$ th symmetric rectangular partial sum $S_{\mathrm{m}}=\Sigma_{|\mathrm{n}| \leqslant \mathrm{m}} c_{\mathrm{n}}$ where $|\mathrm{n}| \leqslant \mathrm{m}$ means $\left|n_{i}\right| \leqslant$ $m_{i}, i=1, \ldots, k$. For a given complex number $s$, consider the relationship

$$
\lim _{n \rightarrow \infty}\left\{\sup _{\mathrm{m} \in N(n, E)}\left|S_{\mathrm{m}}-s\right|\right\}=0 .
$$

If (1) holds for $E=\infty$, we write $U$ - $\lim _{\mathrm{n}} S_{\mathrm{n}}=s$ and say $S$ converges unrestrictedly rectangularly (converges $U$ ) to $s$. If (1) holds for every (arbitrarily large) finite $E, S$ converges restrictedly rectangularly (converges $R$ ) to $s$; and if (1) holds for $E=1$, then $S$ is square convergent to $s$.

A double series $S$ converges circularly to $s$ if $\lim _{R \rightarrow \infty} S_{R}=s$ where $S_{R}=$ $\Sigma_{m^{2}+n^{2}<R^{2}} c_{m n}$ is the $R$ th circular partial sum.

We call a matrix $\sigma_{m \mu}$ row increasing if (a) it is row finite, (b) no row contains the element 0 followed by a nonzero element, and (c) the length of a later row is never less than that of an earlier one; i.e., there is an increasing function $\phi(m)$ such that $\sigma_{m \mu}=0$ if $\mu>\phi(m)$ and $\sigma_{m \mu} \neq 0$ if $\mu \leqslant \phi(m)$.

Let $M_{i}, i=1, \ldots, k$ be one-dimensional linear means with matrices $\left(\left(\sigma_{m \mu}\right)_{i}\right)$. We say that the $k$-fold series $\Sigma c_{\mathrm{n}}$ is summable unrestrictedly rectangularly by the linear method $\bigotimes_{i=1}^{k} M_{i}$-in short, summable $\bigotimes_{i} M_{i}-$ to $s$ if $U \cdot \lim _{\mathrm{n}} \tau_{\mathrm{n}}=$ $s$ where the $\tau_{\mathrm{n}}$ are the rectangular means

$$
\tau_{n}=\sum_{\mu_{1}=0}^{\infty} \sum_{\mu_{2}=0}^{\infty} \ldots \sum_{\mu_{k}=0}^{\infty}\left(\sigma_{n_{1} \mu_{1}}\right)_{1} \cdot\left(\sigma_{n_{2} \mu_{2}}\right)_{2} \ldots\left(\sigma_{n_{k} \mu_{k}}\right)_{k} \cdot c_{\mu_{1}} \ldots \mu_{k} \cdot
$$

The trigonometric series $T(\mathbf{x})=\Sigma c_{\mathrm{m}} \exp i \mathrm{~m} \cdot \mathrm{x}$ has $2^{k}-1$ conjugate trigonometric series $T_{\alpha}$ given by

$$
T_{\alpha}(\mathrm{x})=\sum_{\mathrm{m}} \prod_{j=1}^{k}\left(-i \operatorname{sgn} m_{j}\right)^{\epsilon_{j}} c_{\mathrm{m}} \exp i \mathrm{~m} \cdot \mathbf{x}
$$

where $\alpha=\left(\epsilon_{1}, \ldots, \epsilon_{k}\right), \epsilon_{j} \in\{0,1\}$, not all $\epsilon_{j}=0$.

It is well known that if $T=S[f], f \in L\left(\log ^{+} L\right)^{a}\left(T^{2}\right), a \geqslant 1$, then $T_{(1,0)}=$ $S\left[f_{(1,0)}\right]$ where $f_{(1,0)} \in L\left(\log ^{+} L\right)^{a-1}\left(T^{2}\right)$. (See [12, vol. I, p. 296, problem 6] for the one-dimensional case. This statement follows from that case by integration in the second variable.) The series $T$ is of power series type if $c_{\mathrm{m}}=0$ whenever $\mathrm{m} \notin N(0)$. In this case, all $T_{\alpha}$ are constant multiples of $T\left(T_{\alpha}=\right.$ $\left.(-i)^{\Sigma} \epsilon_{j T}\right)$. 


\section{Counterexamples.}

THEOREM 1. There is a continuous function $H$ on $T^{2}$ such that $S[H]$ is of power series type, has uniformly bounded partial sums, and is everywhere restrictedly rectangularly divergent.

To prove this we require the following lemma.

LEMMA 1. There is a sequence $\left\{P_{n}\right\}$ of trigonometric polynomials of power series type on $T^{2}$ and constants $A, B>0$ such that

(i) $\left|P_{n}(\mathrm{x})\right|<A$ for all $n$ and $\mathrm{x}$;

(ii) $\left|S_{\mathrm{N}}\left[P_{n}\right](\mathbf{x})\right|<A 2^{n}$ for all $n, \mathrm{~N}$, and $\mathrm{x}$; and

(iii) for each fixed pair $E, \mathbf{x}\left(E>1, \mathbf{x} \in T^{2}\right)$,

$$
\limsup _{n \rightarrow \infty}\left\{\sup _{Q \in W(E)}\left|S_{Q}\left[P_{n}\right](\mathbf{x})\right|\right\}>B 2^{n} \text {. }
$$

Assuming temporarily the validity of the lemma, we proceed with the proof of Theorem 1. The required function is

$$
H(\mathrm{x})=\sum_{n=1}^{\infty} \frac{1}{2^{n}} e^{i 1(n) \cdot \mathrm{x}} P_{n}(\mathrm{x})
$$

where the $l(n)=(l(n), l(n))$ are chosen inductively by $l(1)=0$ and $l(n+1)=$ $l(n)+\max \{r, s\}+1$ where $P_{n}(x)=\Sigma_{p, q=0}^{r, s} c_{p q} \exp i(p x+q y)$. This insures that the terms of (2) have pairwise disjoint frequencies in both lattice variables.

Using Lemma 1 (i), we see that the series (2) converges uniformly so that $H$ is continuous on $T^{2}$.

Given $\mathbf{M} \in N(0)$, let $k=\max \{n \mid \mathbf{M}-\mathbf{l}(n) \in N(0)\}$ and $\mathbf{Q}=\mathbf{M}-\mathbf{l}(k)$. Then

$$
\text { (3) } S_{\mathrm{M}}[H](\mathrm{x})=\sum_{n=1}^{k-1} 2^{-n} \exp (i 1(n) \cdot \mathrm{x}) P_{n}(\mathbf{x})+2^{-k} \exp (i 1(k) \cdot \mathbf{x}) S_{\mathrm{Q}}\left[P_{k}\right](\mathbf{x})
$$

which by (i) and (ii) clearly has modulus less than $\sum_{n=1}^{k-1} 2^{-n} A+2^{-k}\left(A 2^{k}\right)<2 A$.

To show divergence $R$ it suffices to show divergence with respect to rectangles of eccentricity at most $E$ for some fixed value of $E>1$. For each point $\mathbf{x}$ of $T^{2}$ there is, by (3) and (iii), a sequence $\left\{\left(n_{i}, \mathbf{Q}_{i}\right)\right\}$ such that $\mathbf{Q}_{i} \in W(E)$ and

$$
\begin{aligned}
& \left|S_{1\left(n_{i}\right)}+Q_{i}[H](x)-S_{1\left(n_{i}\right)-(1,1)}[H](x)\right| \\
& \quad=\left|\frac{1}{2^{n_{i}}} e^{i\left(n_{i}\right) \cdot x} S_{Q_{i}}{ }^{\left[P_{n_{i}}\right](x)}\right|>\frac{1}{2^{n_{i}}} B 2^{n_{i}}=B .
\end{aligned}
$$

Since $1\left(n_{i}\right)+Q_{i} \in W(E)$ (which is a semigroup) and $1\left(n_{i}\right)-(1,1) \in W(E)$, (4) precludes the convergence $R$ of $S[H]$ at $\mathbf{x}$. Since $\mathbf{x}$ was arbitrary, Theorem 1 is proved. 
We proceed to the proof of Lemma 1. The authors have shown [1, Lemmas 1,2 and 4] that there is a family $\left\{h_{\lambda} \mid \lambda \geqslant \lambda_{0}>0\right.$ ) $\}$ of infinitely differentiable functions on $T^{2}$ of power series type satisfying uniformly in $\lambda, x$, and $\mathbf{N}$

(i') $\left|h_{\lambda}(\mathbf{x})\right|<A^{\prime}$;

(ii') $\left|S_{\mathrm{N}}\left[h_{\lambda}\right](\mathbf{x})\right|<A^{\prime} \log \lambda$; and

(iii') $\left|S_{[\lambda y],[\lambda x]}\left[h_{\lambda}\right](x, y)\right|>B^{\prime} \log \lambda$ if $(x, y) \in Q$

for certain positive constants $A^{\prime}, B^{\prime}$ where $Q=[0.1,2 \pi-0.1] \times[0.1,2 \pi-0.1]$ and $[\lambda y]$ denotes the greatest integer in $\lambda y$.

Let $D=\left\{x_{n}\right\}$ be a countable dense subset of $T^{2}$. Let $\lambda(n)=\lambda_{0} \exp \left(2^{n}\right)$ and define $P_{n}$ by

$$
P_{n}(\mathbf{x})=S_{\mathrm{m}(n)}\left[h_{\lambda(n)}\right]\left(\mathbf{x}-\mathbf{x}_{n}\right)
$$

where $\mathrm{m}(n)=(m(n), m(n))$ is chosen with $m(n)$ so large that both $m(n) \geqslant$ $2 \pi \lambda_{0} \exp \left(2^{n}\right)$ and (i) hold with $A=A^{\prime}+1$. The latter choice is possible by (i') since the partial sums of a $C^{\infty}$ function converge uniformly. Property (ii) follows immediately from (ii') and the observation that each partial sum of $P_{n}$ is a partial sum of $h_{\lambda(n)}\left(\mathrm{x}-\mathrm{x}_{n}\right)$.

Now let $\mathrm{x} \in T^{2}$ and $E>1$ be given. Clearly, there is a $\lambda^{\prime}>0$ and a neighborhood $U_{(\pi, \pi)} \subset Q$ such that

$$
\left.W(E) \supset \dot{q}(x, y) \in R_{2} \mid x=\left[\lambda y^{\prime}\right], y=\left[\lambda x^{\prime}\right], \lambda>\lambda^{\prime},\left(x^{\prime}, y^{\prime}\right) \in U_{(\pi, \pi)}\right\} .
$$

Since $\mathbf{x}-D$ is dense, the set $(\mathbf{x}-D) \cap U_{(\pi, \pi)}$ contains an infinite number of points $x-x_{n_{i}}$ such that $\lambda\left(n_{i}\right)>\lambda^{\prime}$. Therefore, the points $Q\left(n_{i}\right)=\left(\left[\lambda\left(n_{i}\right)\left(y-y_{n_{i}}\right)\right]\right.$, $\left.\left[\lambda\left(n_{i}\right)\left(x-x_{n_{i}}\right)\right]\right)$ are in $W(E)$. Since

$$
m\left(n_{i}\right) \geqslant \lambda_{0} \exp 2^{n_{i} \cdot} \cdot 2 \pi>\max \left\{\left[\lambda\left(n_{i}\right)\left(x-x_{n_{i}}\right)\right],\left[\lambda\left(n_{i}\right)\left(y-y_{n_{i}}\right)\right]\right\},
$$

by (iii') we have

$$
\begin{aligned}
\left|S_{\mathrm{Q}_{i}}\left[P_{n_{i}}\right](\mathrm{x})\right| & =\left|S_{\mathrm{Q}_{i}}\left[S_{\mathrm{m}\left(n_{i}\right)}\left[h_{\lambda\left(n_{i}\right)}\right]\right]\left(\mathrm{x}-\mathrm{x}_{n_{i}}\right)\right|=\left|S_{\mathrm{Q}_{i}}\left[h_{\lambda\left(n_{i}\right)}\right]\left(\mathrm{x}-\mathrm{x}_{n_{i}}\right)\right| \\
& >B^{\prime}\left(\log \lambda_{0}+2^{n_{i}}\right),
\end{aligned}
$$

from which (iii) is immediate.

THEOREM 2. There is a function $G(x, y)$ such that $G$ and its three conjugates $G_{\alpha}$ all belong to $L^{1}\left(T^{2}\right)$ and $S[G]$ is unrestrictedly rectangularly convergent almost everywhere, while all the conjugate series are square divergent almost everywhere (despite having unrestrictedly rectangularly bounded partial sums). 
For the proof we shall need the following lemma whose validity is substantially equivalent to the existence of a one-dimensional integrable function of power series type boundedly divergent almost everywhere. (See [12, vol. I, p. 310] where Zygmund proves this refinement of earlier results given by Marcinkiewicz [9] and Kolmogorov [8] .) We omit the proof of the lemma because it is implicitly contained in [12, vol. I, p. 310].

Lemma 2. There is a sequence of power series type trigonometric polynomials on $T^{1}\left\{M_{k}(x)=\Sigma_{\nu=l(k)}^{\mu(k)} c_{\nu} \exp (i v x)\right\}$ where $l(k+1)>\mu(k)$ (pairwise disjoint frequencies) and $l(k)>k$ such that

(i") $\sum_{k=1}^{\infty} k \cdot \int_{0}^{2 \pi}\left|M_{k}(x)\right| d x<\infty$;

(ii") for almost every $x,\left|S_{N}\left[M_{k}\right](x)\right|<A^{\prime \prime}=A^{\prime \prime}(x)$ for all $N$ and $k$; and

(iii") for almost every $x$,

$$
\limsup _{k \rightarrow \infty}\left\{\sup _{q}\left|S_{q}\left[M_{k}\right](x)\right|\right\}>B^{\prime \prime}=B^{\prime \prime}(x)>0 .
$$

Proof OF THEOREM 2. We begin by constructing a function $F$ such that both $F$ and $F_{\beta}=F_{(0,1)}$ are integrable on $T^{2}, S[F]$ converges $U$, while $S\left[F_{\beta}\right]=$ $S_{\beta}[F]$ is square divergent almost everywhere. Let $T \not \equiv 0$ be a Menšov trigonometric series on $T^{1}$ convergent to 0 almost everywhere [12, vol. I, p. 348]. Then the conjugate series $\widetilde{T}$ also converges a.e. (Plessner's theorem) and we must have $\widetilde{T}(x) \neq 0$ a.e. [2, p. 419]. Since the coefficients of $T$ and $\widetilde{T}$ are bounded, an estimate of the partial sums $T_{k}$ and $\widetilde{T}_{k}$ by the sum of the moduli of the coefficients yields

$$
\left|T_{k}\right|<C k, \quad\left|\widetilde{T}_{k}\right|<C k .
$$

Let $F$ be defined by

$$
F(x, y)=\sum_{k=1}^{\infty} M_{k}(x) T_{k}(y) .
$$

By (i") of Lemma 2 and (5) we have

$$
\sum \int_{T_{2}}\left|M_{k}(x) T_{k}(y)\right| d x d y \leqslant \sum 2 \pi C k \cdot \int_{0}^{2 \pi}\left|M_{k}(x)\right| d x<\infty
$$

so that by the Beppo-Levi theorem [10], the series in (6) converges (absolutely and dominatedly) for almost all $(x, y)$ to a well-defined integrable $F$. Similar arguments show that $F_{\beta}$ is integrable and that

$$
\sum\left|M_{k}(x)\right|<C(x)<\infty \text { a.e. }
$$


The dominated convergence and the disjointness of the $M_{k}(x)$ imply that $S[F]$ is obtained by writing out in full the successive polynomials $M_{k}(x) T_{k}(y)$. Observe that all the frequencies of $F$ are lattice points $(m, n)$ satisfying $|n|<m$, i.e., lie "below the diagonal." At almost every point $(x, y)$ relations (7), Lemma 2 (ii") and (iii") hold at $x$, while $T_{k}(y) \rightarrow 0$, and $\widetilde{T}_{k}(y) \rightarrow \widetilde{T}(y) \neq 0$. We fix such a point and simplify notation by suppressing the dependence of partial sums on $x$ and $y$. To prove convergence $U$ of $F=\Sigma M_{k} T_{k}$, let $m$ and $n$ be given. Letting $k^{\prime}=k^{\prime}(m)=\max \{k \mid \mu(k) \leqslant m\}$ and $m^{\prime}=m^{\prime}\left(k^{\prime}(m)\right)=\mu\left(k^{\prime}\right)=$ degree of $M_{k^{\prime}}$, we have

$$
\begin{aligned}
\mid F-S_{m n} & {[F]|\leqslant| F-S_{m^{\prime} n}[F]|+| S_{m^{\prime} n}[F]-S_{m n}[F] \mid } \\
=\left|\sum_{k=k^{\prime}+1}^{\infty} M_{k} T_{k}-\sum_{k=1}^{k^{\prime}} M_{k}\left(S_{n}\left[T_{k}\right]-T_{k}\right)\right|+\left|S_{m}\left[M_{k^{\prime}+1}\right] S_{n}\left[T_{k^{\prime}+1}\right]\right| & +\quad \text { II. }
\end{aligned}
$$

Using the convention that $\Sigma_{i}=0$ if $i>j$, we obtain

$$
\begin{aligned}
I & =\left|\sum_{k=k^{\prime}+1}^{\infty} M_{k} T_{k}-\sum_{k=n+1}^{k^{\prime}} M_{k}\left(T_{n}-T_{k}\right)\right| \\
& \leqslant 3 \sup _{k}\left|T_{k}\right|_{k=\min \left\{n+1, k^{\prime}+1\right\}}\left|M_{k}\right|
\end{aligned}
$$

and

$$
\left.\mathrm{II}=\left|S_{m}\left[M_{k^{\prime}+1}\right]\right| \cdot \mid T_{\min \left\{n, k^{\prime}+1\right.}\right\} .
$$

Now if $(m, n) \in N(p)$ with $p$ very large, then $n, m$, and, consequently, $k^{\prime}$ will all be large. Since $\left\{T_{k}\right\}$ is bounded and $\Sigma\left|M_{k}\right|<\infty$, from (8) we see that $I$ is small. Since $T_{k} \rightarrow 0$ and (ii") of Lemma 2 holds, equality (9) implies that II is also small. The argument for boundedness $U$ of $F_{\beta}$ runs parallel. The terms corresponding to I will still tend to 0 , while the term analogous to II $-\left|S_{m}\left[M_{k^{\prime}+1}\right]\right|$ - $\left|\widetilde{T}_{\min \left\{n, k^{\prime}+1\right\}}\right|-$ is bounded since $\left\{\widetilde{T}_{k}\right\}$ is bounded.

To demonstrate the square divergence of $S_{\beta}[F]$ almost everywhere, let $(k, q)$ be any of the infinite set of integer pairs which satisfy $\left|S_{q}\left(M_{k}(x)\right)\right|>$ $B^{\prime \prime}(x)$ while $\left|\widetilde{T}_{k}(y)\right|>C^{\prime \prime}(y)>0$. Then since $q>q^{\prime}(q)>k^{\prime}(q)=k-1$ and all frequencies of $S_{\beta}[F]$ are lattice points "below the diagonal," we have

$$
\begin{aligned}
\left|S_{\beta_{q q}}[F]-S_{\beta_{q^{\prime} q^{\prime}}}[F]\right| & =\left|\left(S_{\beta_{q q}}[F]-S_{\beta_{q^{\prime} q}}[F]\right)+\left(S_{\beta_{q^{\prime} q}}[F]-S_{\beta_{q^{\prime} q^{\prime}}}[F]\right)\right| \\
& =\left|S_{q}\left[M_{k}\right] \cdot \widetilde{T}_{k}+0\right|>B^{\prime \prime}(x) \cdot C^{\prime \prime}(y)>0 .
\end{aligned}
$$


Since $q>q^{\prime}(q)>k^{\prime}(q)=k-1$ may be arbitrarily large, we see that $S_{\beta}[F]$ cannot square converge at $\mathrm{x}=(x, y)$.

Now let

$$
G_{r s}(x, y)=r F(x, y)+s F(y, x)
$$

be a family of functions indexed by $R^{2}$. Clearly, $G_{r s}$ is convergent $U$ a.e. for every choice of $r$ and $s$. From the previous part of the proof it is clear that at least one term on the right-hand side of $(10)$ is square divergent for each conjugate. Consider, for example, $\beta=(1,1)$. Since

$$
S_{\beta}\left[G_{r s}\right]=-i r S_{(0,1)}[F](x, y)-i s S_{(0,1)}[F](y, x),
$$

it follows that for $s \neq s^{\prime}$

$$
\left(G_{r s}\right)_{\beta}-\left(G_{\left.r s^{\prime}\right)_{\beta}}=-i\left(s-s^{\prime}\right) S_{(0,1)}[F](y, x)\right.
$$

is divergent almost everywhere so that the sets $A_{r s}=\left\{(x, y) \in T^{2} \mid S_{\beta}\left[G_{r s}\right](x, y)\right.$ is square convergent\} satisfy $\left|A_{r s} \cap A_{r s^{\prime}}\right|=0$ if $s \neq s^{\prime}$. Hence, $\left|A_{r s}\right| \neq 0$ for at most countably many $s$ so that, by Fubini's theorem, almost all choices of parameters $(r, s)$ have $\left|A_{r s}\right|=0$. The other two conjugates may be similarly treated so that for almost all choices of parameters $(r, s)$, all three conjugate series are square divergent almost everywhere. Let $G=G_{r s}$ for any such $(r, s)$.

THEOREM 3. There is a continuous function $F$ on $T^{n}, n \geqslant 3$, such that $F$ and all its conjugates $F_{\alpha}$ are continuous, $S[F]$ is unrestrictedly rectangularly convergent almost everywhere, but all $S_{\alpha}[F]$ are restrictedly rectangularly divergent almost everywhere (despite being unrestrictedly bounded a.e.).

Proof. For simplicity, let $n=3$; the general case requires no additional ideas. It will be sufficient to construct a function $H(x, y, z)$ such that $H$ and $H_{(0,0,1)}=H_{\beta}$ are continuous, $S[H]$ converges $U$ a.e., but $S\left[H_{\beta}\right]$ diverges $R$ a.e., since the argument concluding the proof of Theorem 2 generalizes easily to higher dimensions. Define

$$
\begin{aligned}
H(x, y, z) & =\sum_{k=1}^{\infty} \frac{1}{2^{k}} e^{i(k) \cdot(x, y)} P_{k}(x, y) T_{k}(z) \\
& =\sum_{k=1}^{\infty} H_{k}(x, y) T_{k}(z)
\end{aligned}
$$

where $1(k)=(l(k), l(k))$ and $P_{k}$ are as in Lemma 1 , and $T_{k}(z)$ are the partial sums of the Menšov trigonometric series introduced in the proof of Theorem 2. 
The proof that $H$ has the desired properties parallels the discussion of $F$ in the proof of Theorem 2, so we may be brief. The continuity of $H=\Sigma H_{k} T_{k}$ and $H_{\beta}=\Sigma H_{k} \widetilde{T}_{k}$ follows from the uniform convergence of these series (Lemma $1(\mathrm{i})$ and (5)).

Let $k^{\prime}=k^{\prime}(\mathrm{m})=\max \left\{l \in Z \mid \Sigma_{k=1}^{l} H_{k}=S_{(q, q)}\left[\sum_{k=1}^{\infty} H_{k}\right], 0 \leqslant(q, q) \leqslant \mathrm{m}\right\}$ and $\mathrm{m}^{\prime}=\mathrm{m}^{\prime}(\mathrm{m})=\left(m^{\prime}, m^{\prime}\right)$ as a particular choice of $(q, q)$ for which this maximum is attained.

The convergence $U$ of $H$ at points $(x, y, z)$ where $T_{k}(z) \rightarrow 0$ follows from Lemma 1(i) and (ii) and

$$
\begin{aligned}
\left|H-S_{\mathrm{m}, n}[H]\right| & \leqslant\left|H-S_{\mathrm{m}^{\prime}, n}[H]\right|+\left|S_{\mathrm{m}^{\prime}, n}[H]-S_{\mathrm{m}, n}[H]\right| \\
& \left.\leqslant 3 \sup _{k}\left|T_{k}\right| \cdot\left(\sum_{k=\min \left\{n+1, k^{\prime}+1\right\}}^{\infty}\left|H_{k}\right|\right)+\left|S_{\mathrm{m}}\left[H_{k^{\prime}+1}\right]\right| \cdot \mid T_{\min \left\{n, k^{\prime}+1\right.}\right\}
\end{aligned}
$$

as in Theorem 2 (similarly for the unrestricted boundedness of $H_{\beta}$ ).

For divergence of $H_{\beta}$, let $E>1$ be given and fix an $(x, y, z)$ where $\widetilde{T}_{k}=\widetilde{T}_{k}(z) \rightarrow \widetilde{T} \neq 0$. From Lemma 1 (iii) and the definition of $H_{k}$, it is clear that there is an infinite set of pairs $(\mathrm{m}, k)$ with $\mathrm{m} \in W(E)$ such that $\left|S_{\mathrm{m}}\left[H_{k}\right]\right|>$ $B$ while $\left|\widetilde{T}_{k}\right|>C(z)$. For the $\mathrm{m}$ from each of these pairs we have (since, with $\mathrm{m}=$ $(m, n), n \geqslant m^{\prime}>l(k-1)>k-1$ which implies that the frequencies of $H$ satisfy $\left.\left|n_{3}\right|<n_{2}\right)$

$$
\begin{aligned}
\mid\left(S_{\beta}\right)_{\mathrm{m} n}[H] & -\left(S_{\beta}\right)_{\mathrm{m}^{\prime} m^{\prime}}[H] \mid \\
& =\left|\left(\left(S_{\beta}\right)_{\mathrm{m} n}[H]-\left(S_{\beta}\right)_{\mathrm{m}^{\prime} n}[H]\right)+\left(\left(S_{\beta}\right)_{\mathrm{m}^{\prime} n}[H]-\left(S_{\beta}\right)_{\mathrm{m}^{\prime} m^{\prime}}[H]\right)\right| \\
& =\left|S_{\mathrm{m}}\left[H_{k}\right] \cdot S_{n}\left[\widetilde{T}_{k}\right]+0\right|>B C(z)>0 .
\end{aligned}
$$

Therefore, $S_{\beta}[H]$ cannot converge restrictedly for any $E>1$ even if we, in addition, constrain the indices of the partial sums to lie in the plane $n_{2}=n_{3}$.

\section{Positive results.}

THEOREM 4. If $f \in L^{p}\left(T^{2}\right), p>1$ (or even if $f \in L\left(\log ^{+} L\right)^{4}\left(T^{2}\right)$ ), and if $S[f]$ converges unrestrictedly rectangularly on a set $E$, then all three conjugate series $S_{\alpha}[f]$ also converge unrestrictedly rectangularly almost everywhere on $E$.

To prove this we will require Theorems 5 and 6 as follows.

THEOREM 5 (GOGÖLADZE). Let the trigonometric series $S$ be convergent $U$ on $E\left(E \subset T^{2}\right)$. If $S_{(1,0)}=S_{\alpha}$ is summable $(C, 1,0)$ almost everywhere on $E$ to $\widetilde{\sigma}(x, y)$, then $S_{\alpha}$ is convergent $U$ almost everywhere on $E($ to $\widetilde{\sigma}(x, y))$.

For the reader's convenience we will append our proof of this, which was announced by Gogöladze in 1969 [6]. 
THEOREM 6. If $f \in L\left(\log ^{+} L\right)^{2}\left(T^{2}\right)$, then $f$ is summable $(C, 1,0)$ almost everywhere.

This theorem, which was proved in [2, pp. 432-435], is a consequence of the Carleson-Hunt theory of convergence on $T^{1}$.

Proof Of Theorem 4. Let $f \in L\left(\log ^{+} L\right)^{4}$ be such that $S[f]$ converges $U$ on $E$. The function $f_{\alpha}=f_{(0,1)}$ is in $L\left(\log ^{+} L\right)^{3} \subset L\left(\log ^{+} L\right)^{2}$ (see \&2) so that by Theorem $6, S\left[f_{\alpha}\right]$ is summable $(C, 1,0)$ almost everywhere and, in particular, on a full measured subset $E_{1} \subset E$. By Theorem $5, S_{\alpha}[f]=S\left[f_{\alpha}\right]$ converges $U$ on a full measured subset of $E_{1}$ and, hence, almost everywhere on $E$. Symmetrically, $S_{(1,0)}[f]$ converges $U$ a.e. on $E$. Finally, $S_{(1,1)}[f]=$ $S_{(1,0)}\left[f_{(0,1)}\right]$ and $f_{(1,1)} \in L\left(\log ^{+} L\right)^{2}$ so that the convergence of $S_{(1,1)}[f]$ almost everywhere on $E$ follows by an iteration of the above argument.

ProOF OF THEOREM 5. We follow the line of argument used in the proof of Kuttner's one-dimensional theorem [12, vol. I, p. 176]. We may suppose that $|E|>0$, and that $S_{\alpha}$ is summable $(C, 1,0)$ everywhere in $E$. Let $s_{m n}$, $\tilde{s}_{m n}, \sigma_{m n}, \tilde{\sigma}_{m n}$ denote the partial sums and the $(C, 1,0)$ means of $S[f]$ and $S\left[f_{\alpha}\right]$ respectively, and let

$$
\sigma(x, y)=U-\lim \sigma_{m n}(x, y) \text { and } \tilde{\sigma}(x, y)=U-\lim \tilde{\sigma}_{m n}(x, y)
$$

whenever the limits exist.

For $\alpha_{m}=O\left(m^{-1}\right)$ the relation

$$
U-\lim \left\{1 \frac{1}{2}\left[s_{m n}\left(x+\alpha_{m}, y\right)-s_{m n}\left(x-\alpha_{m}, y\right)\right]\right.
$$

$$
\left.+\left[\tilde{s}_{m n}(x, y)-\tilde{\sigma}_{m n}(x, y)\right] \sin m \alpha_{m}\right\}=0
$$

holds for almost every $(x, y) \in E$ and depends on the fact that the expression in curly brackets $-\tau_{m n}(x, y)$-satisfies

$$
\tau_{m n}(x, y)=\sum_{k=1}^{m} a_{m k} \widetilde{\sigma}_{k n}(x, y)
$$

where $\left(a_{m k}\right)$ is the matrix of a linear means (with row sums equal to 0 ). (For details, see [12, vol. I, pp. 74 and 113].)

The proof of (12) requires

$$
\widetilde{\sigma}_{k n}(x, y) \text { converges } U
$$

and

$$
\sup _{k, n} \widetilde{\sigma}_{k n}(x, y) \mid \leqslant B=B(x, y) .
$$


Now (14) was postulated to hold on $E$, but unlike the one-dimensional situation (14) may occur while (15) fails. (The numerical series in [2, p. 406, Remark] is $(C, 1,0)$ summable to zero but has unbounded $(C, 1,0)$ means.) That $(15)$ does indeed hold almost everywhere on $E$ follows from Theorem 7 below (with $k=2,\left(\sigma_{m \mu}\right)_{1}=1-|\mu| /(m+1)$ for $|\mu| \leqslant m, 0$ otherwise, and $\left(\sigma_{m \mu}\right)_{2}=1$ for $|\mu| \leqslant m, 0$ otherwise).

Taking Theorem 7 for granted, we prove that (12) holds on $E$ whenever (15) holds. Fix an $(x, y) \in E$ for which (15) holds and let $\epsilon>0$ be given. Choose $M_{1}=M_{1}(\epsilon)$ so large that $\sup \left\{\left|\tilde{\sigma}_{k n}(x, y)-\tilde{\sigma}(x, y)\right|:(k, n) \in N\left(M_{1}\right)\right\}$ $<\epsilon$. Next choose $M>M_{1}$ so large that $\sum_{k=1}^{M_{1}-1}\left|a_{m k}\right|<\epsilon$ whenever $m \geqslant M$. Then for $(m, n) \in N(M)$,

$$
\begin{aligned}
\tau_{m n}(x, y) & =\sum_{k=1}^{m} a_{m k} \widetilde{\sigma}_{k n}(x, y)=\sum_{k=1}^{m} a_{m k}\left[\widetilde{\sigma}_{k n}(x, y)-\widetilde{\sigma}(x, y)\right] \\
& =\sum_{k=1}^{M_{1}-1}+\sum_{M_{1}}^{m}
\end{aligned}
$$

By (15) and $m \geqslant M$, the first term is $\leqslant 2 B(x, y) \epsilon$, and by the choice of $M_{1}$, the second is $\leqslant\left(\sum_{k=1}^{m}\left|a_{m k}\right|\right) \epsilon$.

Now let $F$ be the full measured subset of $E$ on which (12) holds and let $E \subset F$ be a set of positive measure in which $s_{m n}$ converges $U$-uniformly. (By the theorem of Egerov, which extends in a routine way to convergence $U$, $|F-E|$ can be made arbitrarily small.) In particular, $\sigma(x, y)$ is continuous on $E$. Let $(x, y)$ be a point of $E$ which is also a point of $x$-density of $E$ (i.e., $(2 h)^{-1} \int_{-h}^{h} \chi(x, y) d x \rightarrow 1$ as $h \rightarrow 0$ where $\chi$ is the characteristic function of $E$; the set of such $(x, y)$ has full measure [11, p. 298]). Then for all large enough $m$, there is a number $\mu_{m}, 1 \leqslant \mu_{m} \leqslant 2$, such that $\left(x+\mu_{m} / m, y\right)$ and $\left(x-\mu_{m} / m, y\right)$ both belong to $E$. (See [12, vol. II, p. 176].)

We now apply (12) with $\alpha_{m}=\mu_{m} / m$. By the $U$-uniform convergence of $\left\{s_{m n}\right\}$ on $E$, the first term in square brackets tends to 0 as $(m, n)$ goes unrestrictedly to $(\infty, \infty)$. Observing that $\sin m \alpha_{m}=\sin \mu_{m}$ stays away from zero, we get $U \cdot \lim \left[\tilde{s}_{m n}(x, y)-\tilde{\sigma}_{m n}(x, y)\right]=0$ a.e. on $E$ and, hence, a.e. on $E$.

THEOREM 7. If the linear means $M_{i}=\left(\left(\sigma_{m \mu}\right)_{i}\right), i=1, \ldots, k$, are row increasing and if a trigonometric series $T(\mathbf{x})$ is summable $\bigotimes_{i} M_{i}$ at each $\mathbf{x}$ of a set $E$ of positive measure (more generally, if $\overline{\lim }_{j \rightarrow \infty} \sup _{\mathrm{n} \in N(j)}\left|T_{\mathrm{n}}(\mathbf{x})\right|<\infty$ for $\mathbf{x} \in E)$, then there is a set $F \subset E,|F|=|E|$ such that all rectangular $\bigotimes_{i} M_{i}$ means are bounded on $F$. (The bound may vary from point to point.) In particular, if $E=T^{k}$ (or even if the complement of $E$ is countable), then the conclusion holds everywhere on $E$. 
Proof. The proof is similar to that of Lemma 2.3 of [2, p. 407]. Let $k=2$. Let $F=\left\{\left(x_{0}, y_{0}\right) \in E\right.$ : the linear measures of $E_{x_{0}}=E \cap\left\{x=x_{0}\right\}$ and $E_{y_{0}}=E \cap\left\{y=y_{0}\right\}$ are both positive $\}$. It will suffice to show the $\sigma_{m \mu} \otimes \tau_{n \nu}$ means $T_{m n}$ are bounded at each $\left(x_{0}, y_{0}\right) \in F$. Given $\left(x_{0}, y_{0}\right)$ find $B$ and $q$ so large that

$$
\begin{aligned}
& \left|T_{m n}\left(x_{0}, y\right)\right| \leqslant B \text { whenever } m \text { and } n \geqslant q \text { and } y \in F_{x_{0}}, \\
& \left|T_{m n}\left(x, y_{0}\right)\right| \leqslant B \text { whenever } m \text { and } n \geqslant q \text { and } x \in F_{y_{0}},
\end{aligned}
$$

where $F_{x_{0}} \subset E_{x_{0}}$ and $F_{y_{0}} \subset E_{y_{0}}$ have positive measure. Further choose $B$ so large that $\left|T_{m n}\left(x_{0}, y_{0}\right)\right| \leqslant B$ if $m$ and $n$ are both less than $q$. We still have to study $T_{m n}\left(x_{0}, y_{0}\right)$ when $m \geqslant q>n$ or $n \geqslant q>m$. The two cases are symmetrical; so henceforth, assume $m \geqslant q>n$. From (16), $\left|T_{m q}\left(x_{0}, y\right)\right| \leqslant B$ for all $y \in F_{x_{0}}$. Thinking of $T_{m q}$ as a polynomial in $e^{i y}$ of degree $2 \phi_{2}(q)$, since

$$
\left|T_{m q}\left(x_{0}, y\right)\right|=\left|\sum_{\nu=-\phi_{2}(q)}^{\phi_{2}(q)} \tau_{q|\nu|} \sum_{\mu=-\phi_{1}(m)}^{\phi_{1}(m)} \sigma_{m|\mu|} c_{\mu \nu} e^{i \mu x} 0 e^{i\left(\nu+\phi_{2}(q)\right) y}\right|
$$

and applying a theorem of Paul Cohen [2, Lemma 2.2] which states that for a polynomial $P(z), \sup \left\{|P(z)|: z \in T^{\mathbf{1}}\right\} \leqslant c$ sup $\{|P(z)|: z \in H\}$ where $H \subset T^{\mathbf{1}}$, $|H|>0$, and $c$ depends only on the measure of $H$ and the degree of $P$, we obtain $\left|T_{m q}(x, y)\right| \leqslant B \cdot c$ for all $y$. Since a polynomial is its own Fourier series and a function's supremum dominates its Fourier coefficients, we obtain the same inequality for the coefficients

$$
\left|\tau_{q|\nu|} \sum_{\mu=-\phi_{1}(m)}^{\phi_{1}(m)} \sigma_{m|\mu|} c_{\mu \nu} e^{i \mu x} 0\right| \leqslant B \cdot c, \quad \nu=-\phi_{2}(q), \ldots, \phi_{2}(q) .
$$

Multiplying by $\tau_{n|\nu|} \mid \tau_{q|\nu|}$ and summing over $\nu$ yields

$$
\begin{aligned}
\left|T_{m n}\left(x_{0}, y\right)\right| & \leqslant \sum_{\nu=-\phi_{2}(n)}^{\phi_{2}(n)} \frac{\tau_{n|\nu|}}{\tau_{q|\nu|}} B \cdot c \\
& \leqslant\left(2 \phi_{2}(n)+1\right) \max _{|\nu| \leqslant \phi_{2}(n) ; 0 \leqslant n<q}\left\{\frac{\tau_{n|\nu|}}{\tau_{q|\nu|}}\right\} B \cdot c
\end{aligned}
$$

which, for all $y$ and, hence, for $y=y_{0}$, is bounded since no $\tau_{q|\nu|}=0$ and the number of terms in the max is bounded by $\left(2 \phi_{2}(q)+1\right) \cdot q$. For $k>2$, proceed by induction. 


\section{Circular convergence.}

TheOREM 8. Plessner's theorem does not hold for circular convergence. More precisely, there is a function $g \in L^{1}\left(T^{2}\right)$ such that $S[g]$ converges circularly almost everywhere, while $S_{(0,1)}[g]$ diverges circularly almost everywhere.

Proof. Since unrestricted rectangular convergence implies square convergence, it follows from the proof of Theorem 2 that the function $F(x, y)$ of equation (6) has the property that $S[F]$ is square convergent a.e. while $S_{(0,1)}[F]$ is square divergent a.e. We modify $F(x, y)$ to $g(x, y)=\Sigma e^{i \mu_{k} x} M_{k}(x) T_{k}(y)$ where the $\mu_{k}>0$ are chosen so that disjointness is preserved and are so large that (a) each circular partial sum $S_{R}$ differs from the square partial sum $S_{[R]-1,[R]-1}$ by at most terms whose frequencies lie on the single line $m=$ $[R]$ and so that (b) each square partial sum is identical with some circular partial sum. Then $g$ will have the same properties with respect to unrestricted rectangular convergence and square convergence as $F$. By (b), we see that $S_{(0,1)}[g]$ must diverge by the circular method since $g$ does by the square method. On the other hand, by (a) we have

$$
S_{R}[g](x, y)=S_{[R]-1,[R]-1}[g](x, y)+C_{[R]} e^{i[R] x} S_{l(R)}\left[T_{k(R)}\right](y)
$$

where $k(R)$ is the index of the block sliced by the circle, $l(R) \leqslant$ degree of $T_{k(R)}(y)$, and $C_{[R]}$ is the $[R]$ th Fourier coefficient of $\Sigma e^{i \mu_{k} x} M_{k}(x) \in L^{1}\left(T^{1}\right)$.

Thus, $S[g]$ is circularly convergent a.e. since as $R \rightarrow \infty$ the first term on the right converges a.e. while the second converges to zero a.e. $\left(C_{[R]} \rightarrow 0\right.$ being the Fourier coefficient of an $L^{1}$ function; $\sup _{R}\left|S_{l(R)}\left[T_{k(R)}\right](y)\right| \leqslant \sup _{k}\left|T_{k}(y)\right|$ $<\infty$ a.e. since $T_{k}(y) \rightarrow 0$ a.e.).

6. Remarks.

1. That in higher dimensions Plessner's theorem could not hold in full strength became evident from counterexamples of Gogöladze [6] and the present authors [1]. Gogöladze displayed an almost everywhere convergent double trigonometric series $S$ which is the Fourier series of a function integrable on the torus and whose conjugate series $S_{(0,1)}$ diverges on a set of positive measure, while our example was the Fourier-Stieltjes series of a singular measure on the torus having almost everywhere divergent conjugate. These two examples left open the possibility of a weak form of Plessner's theorem: convergence of a double Fourier series almost everywhere on the torus implies convergence of the conjugate on some subset of positive measure. From Theorem 2 we see this is not so.

2. The "everywhere" character of Theorem 1 stands in marked contrast to the "almost everywhere" nature of the corresponding one-dimensional example 
(which states that there is a function in $H^{1}\left(T^{1}\right)$ with boundedly divergent Fourier series almost everywhere) [12, vol. I, p. 310]. The existence of the example of Theorem 1 is a manifestation of the failure of the Carleson theory in dimension greater than one ([4] and [1]). A one-dimensional "everywhere" example would preclude Carleson's one-dimensional theorem; it was observed by Marcinkiewicz that the existence of an $H^{1}\left(T^{1}\right)$ everywhere divergent series would imply the existence of an $L^{2}$ series divergent on a set of positive measure $[12$, vol. I, p. 314].

3. As an easy corollary of Theorems 1 and 4 and Remark 6 below, one obtains the existence of a real-valued continuous function $f$ such that $f$ and all its conjugates diverge unrestrictedly boundedly almost everywhere. In this connection we note that in [1] the authors showed by explicit calculation that the real part of the function of Fefferman's counterexample [4] had continuous conjugates all of which diverged restrictedly unboundedly everywhere.

4. A triple series $S$ converges by the two parameter method $h$ if there is a positive integer valued function $h$ of two independent positive integer variables $\left(n_{1}, n_{2}\right)$ with $\lim _{n_{1} \rightarrow \infty} h\left(n_{1}, n_{2}\right)=\lim _{n_{2} \rightarrow \infty} h\left(n_{1}, n_{2}\right)=\infty$ such that the limit of $S_{n_{1}, n_{2}, h\left(n_{1}, n_{2}\right)}$ exists as $\left(n_{1}, n_{2}\right)$ tends restrictedly to $(\infty, \infty)$. One can show that the $z$-conjugate of the function $F$ of Theorem 3 diverges a.e. by the two parameter method $h$.

In view of this two parameter nature of the divergence exhibited by $S[F]$ in the proof of Theorem 3, one might ask if a sharper example might be foundfor example, a continuous function on $T^{3}$ whose $z$-conjugate is square divergent on a set of positive measure. This cannot be since the $z$-conjugate would be in $L^{2}$ and thus square convergent almost everywhere [5].

5. The $L\left(\log ^{+} L\right)^{4}\left(T^{2}\right)$ which occurs in Theorem 4 is actually needed only to assure that $S_{(1,1)} \in L\left(\log ^{+} L\right)^{2}$; from the proof it is clear that $L\left(\log ^{+} L\right)^{3}\left(T^{2}\right)$ would suffice for the single conjugates $S_{(1,0)}$ or $S_{(0,1)}$.

Since work in the Carleson-Hunt theory $([3],[7])$ has not yet reached a "borderline"-for example, it is known that $S[f]$ converges if $f \in L\left(\log ^{+} L\right)^{2}\left(T^{1}\right)$, say, and may diverge if $f \in L^{1}$, but the problem for $f \in L\left(\log ^{+} L\right)$ is still openand since Theorem 4 depends on the Carleson-Hunt theory, it is not yet possible to say how much one may eventually be able to weaken the hypothesis of Theorem 4. For example, whether $f \in L\left(\log ^{+} L\right)^{2}\left(T^{2}\right)$ and $S[f] U$-convergent implies $S_{(0,1)}[f] U$-convergent is still open.

6. If in the statement of Theorem 5 one replaces unrestrictedly convergent by unrestrictedly bounded and unrestrictedly $(C, 1,0)$ summable by unrestrictedly $(C, 1,0)$ bounded, the consequent becomes " $S_{(1,0)}$ is unrestrictedly bounded almost everywhere on $E$." If the hypothesis of convergence $U$ of $S$ is replaced 
by convergence $R$, square convergence, or any one parameter method while the hypothesis of unrestricted $(C, 1,0)$ summability is retained, then $S_{(1,0)}$ is again convergent almost everywhere on $E$ with respect to the same method. It remains an open question as to what extent the summability hypothesis can be weakened.

7. If one integrates the moduli of both sides of equation (13) over the torus and lets $(m, n) \rightarrow(\infty, \infty)$ unrestrictedly, one may deduce the following two-dimensional mean Kuttner-type theorem.

THEOREM 9. If $f \in L^{p}, 1 \leqslant p \leqslant \infty, S[f]$ is mean convergent (bounded) $U$ and $S_{\beta}[f]$ is $(C, 1,0)$ mean convergent (bounded) $U$, then $S_{\beta}[f]$ is mean convergent (bounded) $U$.

This theorem has content only for $p=1$ or $\infty$, since for other $p$, mean convergence of the partial sums follows from M. Riesz's theorem. This is the two-dimensional analogue of $[12$, vol. I, p. 268, Theorem 6.14].

\section{REFERENCES}

1. J. M. Ash and L. Gluck, A divergent multiple Fourier series of power series type, Studia Math. 44 (1972), 477-491.

2. J. M. Ash and G. V. Welland, Convergence, uniqueness, and summability of multiple trigonometric series, Trans. Amer. Math. Soc. 163 (1972), 401-436. MR 45 \#9057.

3. L. Carleson, On convergence and growth of partial sums of Fourier series, Acta Math. 116 (1966), 135-157. MR 33 \#7774.

4. C. Fefferman, On the divergence of multiple Fourier series, Bull. Amer. Math. Soc. 77 (1971), 191-195. MR 43 \#5251.

5. - On the convergence of multiple Fourier series, Bull. Amer. Math. Soc. 77 (1971), 744-745.

6. L. D. Gogöladze, The summability of double conjugate trigonometric series, Sakharth. SSR Mecn. Akad. Moambe. 54 (1969), 21-24. MR 40 \#6174.

7. R. A. Hunt, On the convergence of Fourier series, Orthogonal Expansions and Their Continuous Analogues (Proc. Conf., Edwardsville, Ill., 1967), Southern Illinois Univ. Press, Carbondale, Ill., 1968, pp. 235-255. MR 38 \#6296.

8. A. N. Kolmogorov, Une série de Fourier-Lebesgue divergente presque partout, Fund. Math. 4 (1923), 324-328.

9. J. Marcinkiewicz, Sur les séries de Fourier, Fund. Math. 27 (1936), 38-69.

10. F. Riesz and B. Sz.-Nagy, Leçons d'analyse fonctionnelle, 2nd ed., Akad. Kiadó, Budapest, 1953; English transl., Functional analysis, Ungar, New York, 1955. MR 17, 175.

11. S. Saks, Theory of the integral, 2nd rev. ed., Monografie Mat., vol. 7, PWN, Warsaw, 1937; reprint, Dover, New York, 1964. MR 29 \#4850.

12. A. Zygmund, Trigonometric series, 2nd rev. ed., Vols. I, II, Cambridge Univ. Press, New York, 1959. MR 21 \#6498.

DEPARTMENT OF MATHEMATICS, DEPAUL UNIVERSITY, CHICAGO, ILLINOIS 60614 